\title{
Platelet Derived Biomaterials for Therapeutic Use: Review of Technical Aspects
}

\author{
Satyam Arora ${ }^{1} \cdot$ Naveen Agnihotri $^{2}$
}

Received: 27 July 2015/Accepted: 14 March 2016/Published online: 29 March 2016

(c) Indian Society of Haematology \& Transfusion Medicine 2016

\begin{abstract}
Whole blood is composed of both cellular and plasma components, providing a rich source of therapeutic products. Of late, platelet derived biomaterial (platelet rich plasma) consisting of plasma proteins and platelets are increasingly being used for various indications. Protocols for preparation and nomenclature of this biomaterial vary widely amongst authors and are often not well defined. Additionally, they are not uniformly documented in the literature, making results difficult to compare or replicate. In this paper we review the evolution and type of these products available for clinical use. Further we will discuss the scientific rational and technical aspects in preparation of these platelet biomaterials in order to administer them in various fields of medicine.
\end{abstract}

Keywords Platelet rich plasma (PRP) · Osteoarthritis . Cellular therapy $\cdot$ Autologous

\section{Introduction}

Whole blood is a rich source of therapeutic products, having both cellular and plasma components. Cellular component include red blood cell concentrates, platelet concentrates and granulocytes indicated majorly for transfusion. Plasma derivatives are fresh frozen plasma and cryoprecipitates for transfusion as well as medicinal products manufactured

Satyam Arora

satyamarora83@gmail.com

1 Department of Transfusion Medicine, Dr. Ram Manohar Lohia Hospital, New Delhi, India

2 Department of Transfusion Medicine, Fortis Hospital, New Delhi, India from large pools of plasma (typically from thousands of plasma donors) by plasma fractionation. Most blood products are to be administered intravenously but topically used blood derived biomaterials represent a unique group of blood products which may be used in combination with plasma- or cell-derived active components. The sources of these products have special interest as products of autologous origin (self) holds a greater safety index than an allogenic product for therapeutic use.

Recently platelet derived biomaterial consisting of plasma proteins and platelets are increasingly being used for various indications. This has been the most exciting and challenging blood component used for non-transfusional indications over the last decade. The major functions of platelets inside the blood vessel are to prevent acute blood loss \& repair vascular walls. Secretion of intracellular mediators and cytokines from cytoplasmic pools as well as $\alpha$ granules on aggregation release more than 800 different proteins in the surrounding media. These proteins have shown to have a paracrine effect $[1,2]$ on different cell types: myocytes [3], tendon cells [37], mesenchymal stem cells from different origins [8-11], chondrocytes [12-14], osteoblasts [3, 15, 16], fibroblasts [17-19] and endothelial cells [20].

Currently, there are several commercial devices available for the preparation of these platelet biomaterials. The common purpose of all these devices is to fraction whole blood into its component parts to allow isolation of plasma with high concentration of platelets but each of these device functions differently. Specifically, these devices and their protocols differ in their method of isolation (one or two step centrifugation), type of the collecting tube, speed of the centrifuge (resulting in plasma preparations with varying volumes), platelet numbers, quantities of growth factors, and concentrations of residual white and red blood cells. In majority of clinical studies conclusively the efficiency of 
these products cannot be commented upon due to different protocols being used for preparing them. Platelet products are biological biomaterial; hence standardization of the method to produce them is necessary before efficiency of these can be commented upon. We herein review the evolution of these biomaterials with scientific rational that prevailed in development of these as well as the technical aspect behind preparing these products.

\section{Scientific Rationale}

The understanding of blood coagulation cascade and role of platelets leading to tissue regeneration [21] following trauma helps us to understand the therapeutic action of these platelet biomaterials. Platelet-mediated primary hemostasis and the formation of a platelet fibrin clot occlude vascular lesions after trauma. Coagulation factors, growth factors, and cytokines released in the clot by the activated platelets orchestrate complex physiological events leading to tissue repair and regeneration.

The basic cytokines identified in platelets include transforming growth factor- $\beta$ (TGF- $\beta$ ), platelet-derived growth factor (PDGF), insulin-like growth factor (IGF-I, IGF-II), fibroblast growth factor (FGF), epidermal growth factor, vascular endothelial growth factor (VEGF), and endothelial cell growth factor. These cytokines play important roles in cell proliferation, chemotaxis, cell differentiation and angiogenesis.

Bioactive factors like serotonin, histamine, dopamine, calcium and adenosine are present in the dense granules of the platelets [22]. These non-growth factors have fundamental [23] effect on the biologic aspects of wound healing. Histamine and serotonin when released by the platelets increase capillary permeability, which in turn allows inflammatory cells a greater access to the wound site and resultant macrophage activation.

The platelets in a clot, contain several cell adhesion molecules like fibronectin, fibrin and vitronectin. These cell adhesion molecules play a role in cell migration and thus also add to the potential biologic activity of the platelets. The clot itself can also play a role in the wound healing by acting as conductive matrix or "scaffold" upon which cells can adhere and begin the wound healing process. Cocktail of all these platelet associated molecules offer a desired environment for the platelet products to offer therapeutic benefit to the patients.

\section{Type of Products and Their Evolution}

Based on either fibrinogen or platelets as the principle determinant, there are variety of biological products used for various clinical applications (Fig. 1).

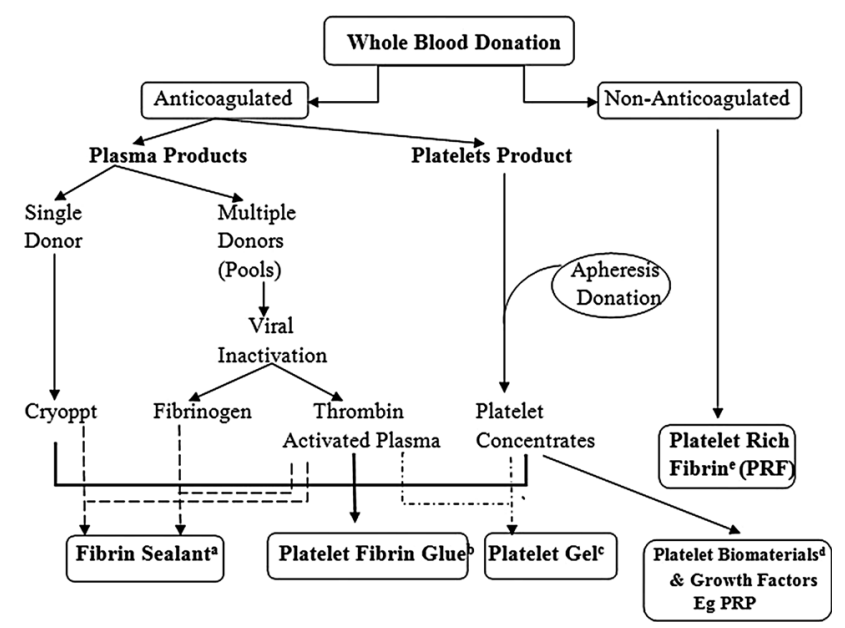

Fig. 1 Variety of biomaterials derived from whole blood

\section{Fibrinogen Based Products}

One of the earliest products used were known as "Fibrin Sealants" (FS). These were one of the initial surgical additives used in Europe during late 1970s. Fibrin Sealant or Fibrin glue or Fibrin tissue adhesives are human plasma derivatives that mimic the final stages of blood coagulation, forming a fibrin clot. These are of two types one is homologous (commercial) FS and the other type is of autologous origin. FS (Fig. 1a) contains concentrated fibrinogen and thrombin as its main active proteins [20], and is usually formulated in the presence of calcium chloride, which speeds up fibrin polymerization. These are mixed to mimic the last stage of coagulation cascade for the therapeutic benefit. They are used for topical hemostasis, tissue sealing and as melting agents for particulate bone substitutes.

\section{Fibrinogen and Platelets Based Products}

Second to follow was a product called "platelet-fibrinogen-thrombin mixtures" or "gelatin platelet-gel foam" [24]. In this new concept, the fibrin glue contained a significant concentration of platelets in the final preparation (Fig. 1b). The motive was to achieve healing from the platelets and to reinforce fibrin clot naturally in the fibrin gel. These new products highlighted the role of platelets in the fibrin gel, and demonstrated promising preliminary results in the fields of ophthalmology, neurosurgery and general surgery. This approach was, for a long time, documented and referred as "platelet-derived wound healing factors or formula-PDWHF", and was successfully used for the treatment of skin ulcers [25]. A platelet gel (Fig. 1c) is obtained by activating platelets by thrombin or $\mathrm{CaCl}_{2}$ to convert fibrinogen into a fibrin gel (platelet gel) and 
activate platelets to release growth factors into the tissue environment [26].

\section{Platelet Based Biomaterials}

In the late 1990s [27, 28] the interest in these platelets based regenerative techniques in oral and maxillofacial surgery gained momentum. Platelet related products at this stage were broadly termed at "platelet rich plasma (PRP)" (Fig. 1d) irrespective of the technique being adapted for its preparation. Soon documenting conceptually and technically similar platelet products under different names created confusion in literature [20].

PRP offered many advantages on FS one of them was being autogenous in origin as it was considered free from risk of HIV \& hepatitis. Additionally immediate preoperative collection of blood for the PRP preparation was a convenient option for the patients. Release of growth factors and cytokines at the site of the interest was not possible by FS whereas it was possible to activate the platelet products using thrombin and thus growth factors could be released at the site for the desired effect. However, the use of bovine thrombin as an activating agent, was a source of concern due to the risk of Creutzfeldt-Jacob disease (mad cow disease). The availability of recombinant thrombin later, however, alleviated this fear.

Choukroun et al. (2001), developed platelet rich fibrin (PRF) in France (Fig. 1e). These were also referred as "second generation" platelet concentrate as they eliminated the use of bovine thrombin. Assigning generation status to this product was not correct as they were part of the family [9] of other related products. In these biomaterial physiological activation of the coagulation cascade during centrifugation lead to the formation of a fibrin clot which could be squeezed into a growth factor-rich fibrin membrane, releasing a cell-free serum-like material (releasate) that contained growth factors and could promote cell growth [29-31].

\section{Platelet Derived Biomaterials: Platelet Rich Plasma (PRP)}

PRP can be defined as a smaller amount of plasma that has a much higher concentration of platelets than in the peripheral blood, achieved by centrifugation of the whole blood [31]. Platelet concentration and amount of growth factors in the PRP depends on the technique used, but on an average, PRP has 3-5 times more platelets and thus growth factors than in the peripheral blood [32].

The collection of whole blood in the presence of an anticoagulant, prevents the initiation of the clotting cascade by blocking the conversion of prothrombin to thrombin [31]. The anticoagulated whole blood is then subjected to single or double centrifugation, depending on the desired character of the final PRP product. PRP biomaterials documented in the scientific literature vary a lot with regards to their preparation especially centrifugation steps.

\section{Factors Determining PRP Preparation}

\section{Venipuncture}

Collection of whole blood for PRP preparation requires great care during venipuncture, in order to avoid any activation of platelets at the phlebotomy site. Venipuncture should be clean without the need to manoeuvre the vein to maintain the flow. Collection should be with a uniform flow and with gentle hands avoiding any activation of platelets. Blood from adult donors can be obtained using a 19-21-gauge needle in a plastic syringe. Some studies do promote collection of blood in an AC primed syringe but the sterility and ratio of $\mathrm{AC}$ to whole blood collected is difficult to maintain.

\section{Volume of Whole Blood}

The volume of whole blood harvested depends on the amount of platelets required in the final product. The normal range of platelets in the whole blood of a healthy individual is between 150,000 and 450,000 platelets $/ \mu \mathrm{L}$ of whole blood. Techniques that harvest more blood will obviously lead to products which contain more platelets. The volume of the whole blood drawn at the beginning should consider the volume of the final product as well as the desired platelet yield (Fig. 4).

\section{Anticoagulant Used}

Anticoagulant (AC) plays an important role in preventing the coagulation cascade of the collected whole blood. Anticoagulant influences the efficacy of the final platelet product in terms of-ratio of anticoagulant to whole blood, rate of collection of whole blood, mixing with the anticoagulant and time between collection of the whole blood and its separation into PRP. The idea is to have minimum aggregation and activation of platelets during anticoagulation of the blood and to harvest maximum naive platelets for therapeutic use. Platelet can undergo alteration during collection, processing and storage which adversely affect their structure and function as well as release of growth factors. Platelet aggregation testing had been utilized for conducting platelet functional studies and effect of AC on platelet function is examined using these testing. 
1. Citrate Sodium citrate at a ratio of nine parts blood to one part anticoagulant is the typical anticoagulant used. Citrate binds the calcium and hence prevents the coagulation cascade. In order to calculate the amount of $\mathrm{AC}$ to be added there is a need to correct for the hematocrit of the whole blood used.

$$
\begin{aligned}
& {[\text { Whole blood to be added to } 1.0 \mathrm{~mL} \text { anticoagulant }} \\
& =5 /(1-0 . H c t)]
\end{aligned}
$$

2. Heparin Heparin inhibits the generation and activity of thrombin via its complex with antithrombin III. "Spontaneous" aggregation of platelets in presence of heparin is seen in some individuals.

3. EDTA Sodium and potassium of EDTA (ethylenediaminetetra-acetic acid) are powerful anticoagulants, which act by chelation of calcium molecules in the blood. EDTA also appears to suppress platelet degranulation hence it is not a recommended AC for platelet biomaterial preparation [33].

4. ACD/ACD-A ACD brings the $\mathrm{pH}$ of the PRP to 6.5, and is, therefore, unsuitable for use in aggregation. The addition of citrate to the blood also makes it more acidic than is physiologic. In anticoagulant citrate dextrose-A (ACD-A), the citrate binds calcium and prevents coagulation, whereas the dextrose and other ingredients support platelet metabolism and viability. This formulation of ACD-A keeps the $\mathrm{pH}$ of the PRP at 7.2. Since some cellular growth factors are influenced by the $\mathrm{pH}$ of the tissue, some protocols recommend buffering the PRP back to a physiologic range prior to injection. ACD is one of the most common AC used by the commercial kits for preparing PRP.

5. CPD/CPDA-1 Citrate phosphate dextrose is similar to ACD-A but has fewer supportive ingredients and may therefore be less effective at maintaining platelet viability. The concentration of citrate in CPD plasma is usually $20-22 \mathrm{mM}$ and used in the ratio of 1:7 with the whole blood. Dextrose provides a source of energy and phosphate serves as a buffer. Adenine, which is added to blood collection bags to improve red cell survival during storage by increasing cellular ATP levels, does not appear to enhance platelet survival during storage. By maintaining $\mathrm{pH}$ levels and concentrations of calcium ions lower than in the physiologic state, platelets are less likely to become activated, release their intracellular contents, and undergo irreversible aggregation.

\section{Role of $\mathrm{pH}$}

As per platelet aggregation studies, platelets are $\mathrm{pH}$ sensitive and, therefore, when preparing the biomaterials
$\mathrm{pH}$ must be maintained between 7.2 and 8.0 [51, 52]. If the $\mathrm{pH}$ of the plasma drops below 6.4, no aggregation will occur and at a $\mathrm{pH}$ above 8.0, spontaneous aggregation can occur. If the $\mathrm{pH}$ approaches 10 , inhibition of aggregation once again is evident. The change in $\mathrm{pH}$ of the plasma is mediated by the diffusion of $\mathrm{CO}_{2}$ out of the plasma. As the $\mathrm{CO}_{2}$ diffuses out of the plasma, the $\mathrm{pH}$ rises. To avoid this situation, PRP should be kept in a tube that minimizes the surface area exposed to the atmosphere (small diameter tubes) and the tube should be kept capped.

\section{Centrifugation}

The basic principle involved in the preparing any PRP biomaterial is the selective separation of the liquid and solid components of whole blood through the technique of centrifugation. Centrifugation technique uses the physical principle described by "Stoke Law", which states that the settling velocity of particles in a liquid environment in response to gravitational forces is approximately proportional to their diameter. Therefore, particles with a larger diameter such as red blood cells ( $\sim 7$ microns in diameter) and WBCs (7-15 microns in diameter) will settle proportionally faster than platelets $(\sim 2$ microns in diameter) when subjected to a gravitational force.

During the process of centrifugation, the movement of the particle is a result of the acting centrifugal force in the radial direction - the gravitational force in the downward direction, and the drag force in the opposing direction of the particle motion. The magnitude of the acting centrifugal force depends on the apparent mass of the particle (corrected to the buoyancy), the angular velocity and its distance from the axis of the centrifugal head or rotor. The greater the distance from the rotor, the greater is the centrifugal force acting on the particle.

In the case of WB the centrifugal force and time drives the packing of erythrocytes at the bottom layer, the volume of plasma at the upper layer, and the recovery efficiency of platelets. According to the size of the commercial tubes assayed, the distances between the surface of WB and the rotor were 4.9 and $3.0 \mathrm{~cm}$ for the processed volumes 3.5 and $8.5 \mathrm{~mL}$, respectively. Therefore for the same angular velocity, the mean centrifugal force applied on the erythrocytes decreased with the smaller mean distance from the rotor for the larger volume $(8.5 \mathrm{~mL})$ processed. These factors explain the decrease of the packing of erythrocyte at the bottom layer and also the recovery efficiency of platelets at the upper layer.

In order to restore the same separation efficiency, the packing of erythrocytes must be restored by an increase in time and centrifugal acceleration. This aspect is also 
relevant when different volumes are transferred from the first to the second spin. In this case, for the same parameters of centrifugation, the remaining amount of platelets in PPP varies due to the changes of the centrifugal force acting on the platelets. As a consequence, the platelet concentration factor is also altered.

The three major variables which affect the recovery of cells from WB by differential centrifugation are rotor size (R), centrifuge speed (RPM), and duration of centrifugation. Many published papers often refer to relative centrifugation force ( $g$ force/RCF) that is derived from the radius of the centrifuge rotor and the revolutions of the rotor (Fig. 2). For a given centrifuge, the rotor size is generally not variable. Therefore, the other two variables (centrifuge speed and duration) can be altered in a stepwise fashion in a simplex strategy to determine the optimal conditions for preparing PRP [34].

Depending on the gravitation force applied (based on the centrifugal force and time) there is selective settling down of the cellular components of the whole blood. A high force (hard spin) will cause settling down of all the cellular components and will yield only plasma in the supernatant whereas a relatively lower force (soft spin/light spin) will allow platelets to remain selectively suspended in the plasma component of blood, whereas the other solid particles (red and WBCs) settle more rapidly. Hence type of centrifugation offered plays a major role in preparing platelet products (discussed later).

Common methods used in blood banks for preparing platelet products for intravenous use may involve single or double spin methods depending on the bag used for blood collection. Broadly there are four type of platelet products prepared based on the spin method used. They include platelet rich plasma (PRP), Buffy-Coat platelet rich plasma (BC-PRP), platelet rich plasma derived Platelet Concentrates (PRP-PC) \& Buffy-Coat derived Platelet Concentrate (BC-PC).

Buffy-Coat is a layer which is between plasma (above) and red cells (below). This layer is formed when an appropriate amount of centrifugal force (stronger enough to settle all the platelets) to the whole blood resulting settling down of all the platelets and leucocytes above the packed red cells. It's a whitish layer formed on the packed red blood cells (PRBC). Based on number of spin required to prepare platelets we could classify their method of preparation as single spin (primary segregation) and double spin (secondary segregation) (Fig. 3).

\[ \mathrm{RCF}=28.38 \times \mathrm{R} \times(\mathrm{RPM} / 1000)^{2} \]
$\mathbf{R C F}=$ relative centrifugal force $(\mathrm{x} g) ; \mathbf{R}=$ radius in inches; $\mathbf{R P M}=$ revolutions per minute

Fig. 2 Formula to calculate centrifugal force

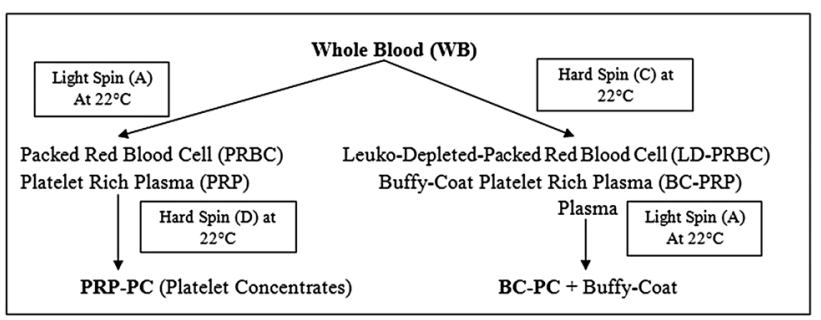

Fig. 3 Number and type of centrifugal spins required to prepare different type of platelet biomaterials

\section{a. Single Spin (Primary Segregation)}

Primary Segregation of the collected whole blood will only prepare platelet rich plasma (PRP). Depending on the type of spin (Light/Hard) it would result in following types of platelet products:

\section{Platelet rich plasma (PRP)}

This is a straw coloured suspension of platelets in plasma prepared when an initial light spin is used for primary segregation of whole blood. Volume of PRP prepared will depend on the initial whole blood collected, hematocrit of the WB and the centrifugal force applied. This is rich in platelets and leukocytes and will ideally have no red cell contamination. The number of platelets in PRP or the platelet yield in PRP product will depend on the centrifugal force applied.

2. Buffy-Coat platelet rich plasma (BC-PRP)

This is a yellow coloured suspension consisting of platelets, leukocytes and red blood cells prepared when initial hard spin is used for primary segregation of whole blood. The centrifugal force will allow all the platelets \& leukocytes to collect on top of the RBC layer resulting in platelet poor plasma on the top. The BC-PRP will contain enriched platelets and leukocytes with red cells as well.

\section{b. Double Spin (Secondary Segregation)}

Secondary Segregation of the platelet rich suspensions prepared will prepare platelet concentrates (PC). Depending on the type of initial segregation (first spin) the subsequent segregation would result in following types of platelet products:

1. platelet rich plasma-platelet concentrates (PRP-PC) PRP when given a hard spin for secondary segregation will yield PRP-PC. This is merely concentrating all the platelets in the PRP into a platelet pallet at the bottom of the tube and PPP (Platelet Poor Plasma) at the top of it. Now depending on how much PPP is aspirated from the top; the resultant PRP-PC with desired volume and concentration can be achieved. This procedure can produce very high concentration of platelets and leucocytes in the final product in a very low volume. 


\section{Buffy-Coat Platelet Concentrates (BC-PC)}

BC-PRP when given a light spin for secondary segregation will yield BC-PC. Due to a light spin the platelets will be suspended in the supernatant plasma and the red cells (which were present in BC-PRP) will be settled. This process yields relatively more yield of platelets in the final product when compared to PRPPC if similar initial volume is used.

Preparation of PRP using a laboratory centrifuge involves functional calibration of the centrifuge for both the preparation of platelet-rich plasma (PRP) from whole blood and subsequent preparation of platelet concentrates (PC) from PRP.

\section{Platelet Count}

The higher number of platelet in the platelet product can proportionately produce a higher degree of clinical response. However, in vitro studies have also shown that the dose-response curves of most growth factors are not linear i.e., at a higher concentration when the cell surface receptors for a specific growth factor are occupied further increasing concentrations of the growth factor have no additional effect. Few authors also suggest that some growth factors at a higher concentration can actually exert an inhibitory effect on cell functions [35, 36].

The dose response relationship is dependent on-concentrations of growth factors in the final product and number of receptors on the response cell type hence a precise concentration of platelet (and thus their associated growth factors) required to optimize the response on the cell types involved is still not clear. The final platelet concentration of any platelet product should be based on (1) the initial volume of whole blood taken (2) the platelet capture/recovery efficiency of the technique used, and (3) the final volume of plasma utilized to suspend the concentrated platelets in cases using two spin protocols. Figure 4 discusses various formulas and terms which can be used to enumerate platelets in the final product or biomaterial in order to assess their clinical efficiency.

\section{Inclusion of $W B C$}

Inclusion of WBCs in the final platelet biomaterial with their effects on the clinical efficacy of platelets is widely debated and is of significant interest to clinicians. The concentration of leukocytes in whole blood ranges from 4 to $11 \times 10^{9} / \mathrm{mL}$. Some authors have recommended against the inclusion of WBCs in PRP to avoid an inflammatory reaction between WBCs and the exposed tissue, whereas others have reported the benefit of increased antibacterial and immunological resistance [37, 38].

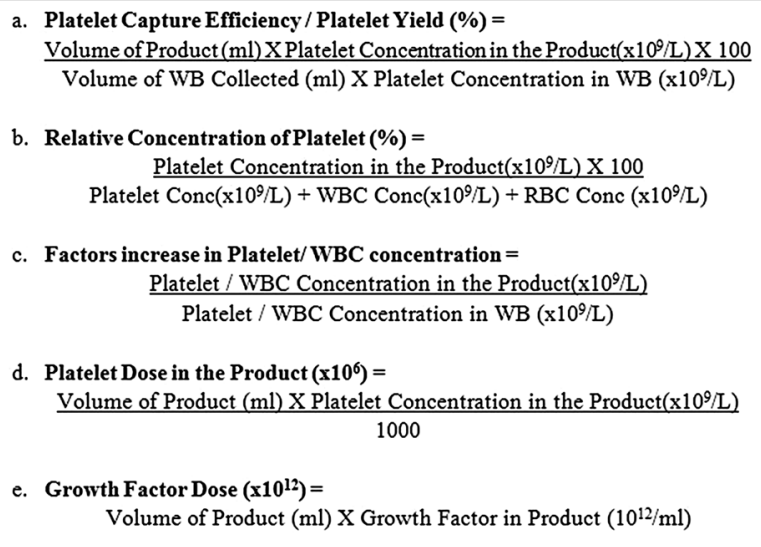

Fig. 4 Formula's and terms which could be used for better reporting of the dose of platelet biomaterials

Leukocytes offers many advantages as they produce VEGF having antimicrobial properties as well as promoting tissue repair process [39-41]. Neutrophils are a main defence against pathogens and initiate a local inflammatory response to prevent infections. Neutrophils release a number of molecules including proteases and reactive oxygen species to destroy microbes, but an excess of these molecules in the absence of an infection may harm uninjured tissue [42]. The antimicrobial properties of WBCs are important as injection related infections are not particularly common but their presence in PRP may be a potential prophylactic measure in joint replacement or other surgical procedures [39]. The immunomodulatory capability of WBCs may also prevent or control the spread of infection at the site of injection, as well as enhance PRP growth factor concentration.

In contrast to the advantageous properties of leukocyterich PRP, a major disadvantage is possible increased inflammation at the site of injection. There is a possibility that PRP products containing WBCs may induce more local pain than PRP preparations without WBCs [43].

As per the American Association of Blood Bank (AABB) any blood component with leukocyte count of $<5 \times 10^{6} / \mu \mathrm{L}$ is regarded as leucoreduced product (LR). This is an internationally accepted criteria which could be adopted to define leucoreduction in platelets biomaterial as well. The lack of certainty regarding the effects of leukocyte-poor versus leukocyte-rich products demands further investigations to develop a more advanced understanding of the benefits and disadvantages of including WBCs in these biomaterials [44].

Therapeutic efficiency of leukocytes in the final products, therefore cannot be commented upon. It is possible that a positive or negative effect of WBC's cannot be generalized to all tissues and all clinical conditions hence 
these preparations containing WBCs may be more beneficial in certain conditions.

\section{Activation of PRP}

In-vivo circulating platelets have a life span of 7 days and their cytokine contents remain within their granules until some event triggers platelet activation and the subsequent secretion of these factors. This concept has been utilized by many authors in order to achieve the desired growth factor levels at the site of interest. Platelet activation can be initiated by a number of methods. Even shear forces caused by fluid flow can cause activation. Contact with a variety of materials including fibrillar collagen, basement membranes of cells and thrombin causes activation and degranulation of these cells. Even double freeze thaw activation of the PRP causes membrane disruption due to temperature shock and release the intracellular cytokines. Sonker et al. [45] showed that $\mathrm{CaCl}_{2}$-induced degranulation technique for platelet activation yielded significantly higher amounts of PDGF-AB and PDGF-BB when compared with double freeze thaw method which yielded higher amounts of IGF1 and TGF- $\beta$.

Some PRP preparation techniques advocate the activation of platelets before injection on the assumption that the bioactive factors contained in the $\alpha$ granules will be secreted and readily available [29]. These protocols rely on bovine or autologous thrombin with or without calcium chloride to activate the platelets and catalyze the fibrinogen to fibrin reaction that begins to congeal the plasma solution [46].

When inactivated PRP is injected into connective tissues it comes into contact with collagen and tissue thromboplastin (tissue factor) that can activate platelets. Therefore, the need for platelet activation with exogenous thrombin before injection is not clear. A recent study has also suggested that activation of PRP with exogenous thrombin may, in fact, be detrimental to the biological activity of the growth factors [47]. In this in vivo study, platelet activation with high levels of exogenous thrombin resulted in a massive release of thrombin from the PRP preparation which, in turn, significantly diminished the biological effect of the thrombin-activated PRP compared with the non activated PRP. As discussed previously, the dose-response curves of growth factors are not linear and may, in fact, be inhibitory at higher concentrations.

It is also important to understand that once activated, platelets begin secreting their growth factors immediately [14]. Approximately $70 \%$ of these growth factors are secreted within the first 10 min after activation and within an hour almost $100 \%$ have been secreted [14]. Because the half life of most growth factors is measured in minutes to hours, activating PRP with exogenous thrombin may
Table 1 Optimal PRP product for various clinical applications

\begin{tabular}{ll}
\hline $\begin{array}{l}\text { Target tissue (clinical } \\
\text { application) }\end{array}$ & Optimal PRP product \\
\hline $\begin{array}{l}\text { Collagen synthesis [53, } \\
54]\end{array}$ & $\begin{array}{l}\geq 10^{6} \text { platelet/ } \mu \mathrm{L} \text { or three to four times of } \\
\text { whole blood } \\
\text { Lack of } \mathrm{WBC} \text { would slow down healing } \\
\text { due to release of pro-inflammatory } \\
\text { factors } \\
\text { Lack of RBC as on lysis of RBC will } \\
\text { release free radicals } \\
\geq 10^{6} \text { platelet/ } \mu \mathrm{L} \text { or three to four times of } \\
\text { whole blood }\end{array}$ \\
$\begin{array}{l}\text { Dermatological } \\
\text { applications [55] }\end{array}$ \\
$\begin{array}{l}\text { Dentistry applications } \\
\text { [56] }\end{array}$ \\
\hline
\end{tabular}

actually shorten the exposure of target cells to these growth factors compared with non thrombin treated PRP. Many studies [48-50] showed that platelets derived biomaterials lose a significant proportion of their PDGF during storage and thus became less efficient in their growth-promoting activity, hence application of these products soon after preparation is recommended.

\section{Platelet Rich Fibrin (PRF)}

PRF preparation is simple product as it involves centrifugation of whole blood, without anticoagulation, at nearly $400 \mathrm{~g}$ for $10 \mathrm{~min}$. The absence of anticoagulant implies the activation in a few minutes of most platelets of the blood sample and release of the coagulation cascades. Fibrinogen is initially concentrated in the high part of the tube, before the circulating thrombin transforms it into fibrin. A fibrin clot is then obtained in the middle of the tube, just between the red corpuscles at the bottom and acellular plasma at the top. The fibrin will polymerize in a diffuse way in the tube and only a small blood clot without consistency will be obtained.

Biochemically PRF consists of assembly of cytokines, glycanic chains, and structural glycoproteins in the mesh of slowly polymerized fibrin network. Due to the presence of platelets it also offers stimulation of defence mechanism along with the regulation of the inflammatory process. Fibrin matrix offers natural guide for angiogenesis and wound healing.

It does not require the use of bovine thrombin or any anticoagulants hence it is completely safe where as a standardized method offers uniform product for clinical applications. As a limitation it offers limited amount of volume when compared with PRP. Tissue banking of these products is not feasible due to short shelf life of PRF and inability to use it as an allogenic graft due to presence of immunogenic cells in the matrix specific to the donor. 
Platelet rich plasma is applicable for various clinical applications such as dermatology, musculo-skeletal wound healing and dentistry. Type of platelet biomaterial, ideally, to be used for each of these applications has still not evolved. Table 1 discusses the characteristics of an optimal platelet rich plasma recommended for these clinical applications.

\section{Conclusion}

In this review we have discussed the evolution and type of these platelet derived biomaterials. We have tried to emphasize on compositions of these products and how they differ with each other. Our attempt was to highlight the variety of different products which could be prepared based on different protocols used to separate them from the whole blood. Therapeutic efficiency of platelet derived biomaterials is still in its initial phase of evaluation in various clinical applications.

In order to compare the clinical efficiency there is a need for quality control of the products used. Standardization with regards to preparation, composition and nomenclature of each type of platelet biomaterial is need of the hour. As it will be essential to, finally demonstrate these biomaterials ability to reproduce consistent clinical results.

\section{References}

1. Senzel L, Gnatenko DV, Bahou WF (2009) The platelet proteome. Curr Opin Hematol 5:329-333

2. Macaulay IC, Carr P, Gusnanto A et al (2005) Platelet genomics and proteomics in human health and disease. J Clin Invest 115:3370-3377

3. Mazzocca AD, McCarthy MB, Chowaniec DM et al (2012) The positive effects of different platelet-rich plasma methods on human muscle, bone, and tendon cells. Am J Sports Med 40:1742-1749

4. de Mos M, van der Windt AE, Jahr H et al (2008) Can plateletrich plasma enhance tendon repair? A cell culture study. Am J Sports Med 36:1171-1178

5. Jo CH, Kim JE, Yoon KS, Shin S (2012) Platelet-rich plasma stimulates cell proliferation and enhances matrix gene expression and synthesis in tenocytes from human rotator cuff tendons with degenerative tears. Am J Sports Med 40:1035-1045

6. Carofino B, Chowaniec DM, McCarthy MB et al (2012) Corticosteroids and local anesthetics decrease positive effects of platelet-rich plasma: an in vitro study on human tendon cells. Arthroscopy 28:711-719

7. Visser LC, Arnoczky SP, Caballero O et al (2010) Growth factorrich plasma increases tendon cell proliferation and matrix synthesis on a synthetic scaffold: an in vitro study. Tissue Eng Part A 16:1021-1029

8. Cho HS, Song IH, Park SY et al (2011) Individual variation in growth factor concentrations in platelet-rich plasma and its influence on human mesenchymal stem cells. Korean J Lab Med $31: 212-218$
9. Dohan Ehrenfest DM, Doglioli P, de Peppo GM et al (2010) Choukroun's platelet-rich fibrin (PRF) stimulates in vitro proliferation and differentiation of human oral bone mesenchymal stem cell in a dose-dependent way. Arch Oral Biol 55:185-194

10. Mishra A, Tummala P, King A et al (2009) Buffered platelet-rich plasma enhances mesenchymal stem cell proliferation and chondrogenic differentiation. Tissue Eng Part C Methods 15:431-435

11. Xie X, Wang Y, Zhao C et al (2012) Comparative evaluation of MSCs from bone marrow and adipose tissue seeded in PRPderived scaffold for cartilage regeneration. Biomaterials 33:7008-7018

12. Drengk A, Zapf A, Stürmer EK, Stürmer KM, Frosch KH (2009) Influence of platelet-rich plasma on chondrogenic differentiation and proliferation of chondrocytes and mesenchymal stem cells. Cells Tissues Organs 189:317-326

13. Spreafico A, Chellini F, Frediani B et al (2009) Biochemical investigation of the effects of human platelet releasates on human articular chondrocytes. J Cell Biochem 108:1153-1165

14. van Buul GM, Koevoet WL, Kops N et al (2011) Platelet-rich plasma releasate inhibits inflammatory processes in osteoarthritic chondrocytes. Am J Sports Med 39:2362-2370

15. Graziani F, Ivanovski S, Cei S et al (2005) The in vitro effect of different concentrations on osteoblasts and fibroblasts. Clin Oral Implants Res 17:212-219

16. García-Martínez O, Reyes-Botella C, Díaz-Rodríguez L et al (2012) Effect of platelet-rich plasma on growth and antigenic profile of human osteoblasts and its clinical impact. J Oral Maxillofac Surg 70:1558-1564

17. Anitua E, Sánchez M, del Mar Zalduendo M et al (2009) Fibroblastic response to treatment with different preparations rich in growth factors. Cell Prolif 42:162-170

18. Kushida S, Kakudo N, Suzuki K, Kusumoto K (2013) Effects of platelet-rich plasma on proliferation and myofibroblastic differentiation in human dermal fibroblasts. Ann Plast 71(2):219-224

19. Browning SR, Weiser AM, Woolf N et al (2012) Platelet-rich plasma increases matrix metalloproteinases in cultures of human synovial fibroblasts. J Bone Joint Surg Am 94:1-7

20. Radosevich M, Goubran HA, Burnouf T (1997) Fibrin sealant: scientific rationale, production methods, properties, and current clinical use. Vox Sang 72:133-143

21. Mann KG (1999) Biochemistry and physiology of blood coagulation. Thromb Haemost 82:165-174

22. Gay LJ, Felding-Habermann B (2011) Contribution of platelets to tumour metastasis. Nat Rev Cancer 11(2):123-134

23. Arai M, Ogita-Nakanishi H, Lee K et al (2012) Role of cytokines in lavage or drainage fluid after hemithyroidectomy in wound healing: involvement of histamine in the acceleration and delay of wound healing. Wound Repair Regen 20(2):158-165

24. Rosenthal AR, Egbert PR, Harbury C, Hopkins JL, Rubenstein E (1978) Use o platelet fibrinogen-thrombin mixture to seal experimental penetrating corneal wounds. Albrecht Von Graefes Arch Klin Exp Ophthalmol 207:111-115

25. Knighton DR, Ciresi KF, Fiegel VD, Austin LL, Butler EL (1986) Classification and treatment of chronic nonhealing wounds. Successful treatment with autologous platelet-derived wound healing factors (PDWHF). Ann Surg 204:322-330

26. Blair P, Flaumenhaft R (2009) Platelet alpha-granules: basic biology and clinical correlates. Blood Rev 23:177-189

27. Marx RE, Carlson ER, Eichstaedt RM, Georgeff KR et al (1998) Platelet-rich plasma: growth factor enhancement for bone grafts. Oral Surg Oral Med Oral Pathol Oral Radiol Endod 85:638-646

28. Whitman DH, Berry RL, Green DM (1997) Platelet gel: an autologous alternative to fibrin glue with applications in oral and maxillofacial surgery. J Oral Maxillofac Surg 55:1294-1299 
29. Su CY, Kuo YP, Tseng YH, Su CH, Burnouf T (2009) In vitro release of growth factors from platelet-rich fibrin (PRF): a proposal to optimize the clinical applications of PRF. Oral Surg Oral Med Oral Pathol Oral Radiol Endod 108:56-61

30. Burnouf T, Lee CY, Luo CW et al (2012) Human blood-derived fibrin releasates: composition and use for the culture of cell lines and human primary cells. Biologicals 40:21-30

31. Marx RE (2001) Platelet-rich plasma (PRP): what is PRP and what is not PRP? Implant Dent 10(4):225-228

32. Foster TE, Puskas BL, Mandelbaum BR, Gerhardt MB, Rodeo SA (2009) Platelet rich plasma: from basic science to clinical applications. Am J Sports Med 37(11):2259-2272

33. Aster Richard H (2013) Blood platelet kinetics and platelet transfusion. J Clin Invest 123(11):4564-4565

34. Reiss RF, Katz AJ (1976) Optimizing recovery of platelets in platelet-rich plasma by the simplex strategy. Transfusion $16: 370-374$

35. Mooren R, Hendriks EJ, van der Beuken J et al (2010) The effect of platelet-rich plasma in vitro on primary cells: rat osteoblastlike cells and human endothelial cells. Tissue Eng Part A 16:3159-3172

36. Ranly DM, McMillan J, Keller T et al (2005) Platelet-derived growth factor inhibits demineralized bone matrix-induced intramuscular cartilage and bone formation. A study of immunocompromised mice. J Bone Joint Surg Am 87:2052-2064

37. Moojen DJ, Everts PA, Schure RM et al (2008) Antimicrobial activity of platelet-leukocyte gel against Staphylococcus aureus. J Orthop Res 26:404-410

38. Mazzocca AD, McCarthy MB, Chowaniec DM et al (2012) Platelet rich plasma differs according to preparation method and human variability. J Bone Joint Surg Am 94:308-316

39. Moojen DJ, Everts PA, Schure RM et al (2008) Antimicrobial activity of platelet-leukocyte gel against Staphylococcus aureus. J Orthop Res 26:404-410

40. Castillo TN, Pouliot MA, Kim HJ et al (2011) Comparison of growth factor and platelet concentration from commercial platelet-rich plasma separation systems. Am J Sports Med 39:266-271

41. Werther K, Christensen IJ, Nielsen HJ (2002) Determination of vascular endothelial growth factor (VEGF) in circulating blood: significance of VEGF in various leucocytes and platelets. Scand $\mathbf{J}$ Clin Lab Invest 62:343-350

42. Lopez-Vidriero E, Goulding KA, Simon DA et al (2010) The use of platelet-rich plasma in arthroscopy and sports medicine: optimizing the healing environment. Arthroscopy 26:269-278
43. Mei-Dan O, Mann G, Maffulli N (2010) Platelet-rich plasma: any substance to it? Br J Sports Med 44:618-619

44. DeLong JM, Russell RP, Mazzocca AD (2012) Platelet-rich plasma: the PAW classification system. Arthroscopy 28:998-1009

45. Sonker A, Dubey A (2015) Determining the effect of preparation and storage: an effort to streamline platelet components as a source of growth factors for clinical application. Transfus Med Hemother 42:174-180

46. Mann KG (1999) Biochemistry and physiology of blood coagulation. Thromb Haemost 82:165-174

47. Han B, Woodell-May J, Ponticiello M et al (2009) The effect of thrombin activation of platelet rich plasma on demineralized bone matrix osteoinductivity. J Bone Joint Surg Am 91:1459-1770

48. Ledent E, Wasteson A, Berlin G (1995) Growth factor release during preparation and storage of platelet concentrates. Vox Sang 68:205-209

49. Wadhwa M, Seghatchian MJ, Lubenko A, Contreras M, Dilger P, Bird C, Thorpe R (1996) Cytokine levels in platelet concentrates: quantitation by bioassays and immunoassays. $\mathrm{Br} \mathrm{J}$ Haematol 93:225-234

50. Fujihara M, Ikebuchi K, Wakamoto S, Sekiguchi S (1999) Effects of filtration and gamma radiation on the accumulation of RANTES and transforming growth factor- $\beta 1$ in apheresis platelet concentrates during storage. Transfusion 39:498-505

51. Murphy S (1985) Platelet storage for transfusion. Semin Hematol 22:165-177

52. Liu Y, Kalén A, Risto O, Wahlström O (2002) Fibroblast proliferation due to exposure to a platelet concentrate in vitro is $\mathrm{pH}$ dependent. Wound Repair Regen 10(5):336-340

53. McCarrel TM, Minas T, Fortier LA (2012) Optimization of leukocyte concentration in platelet-rich plasma for the treatment of tendinopathy. J Bone Joint Surg Am 94(19):e143(1-8)

54. Weibrich G, Kleis WK, Hitzler WE, Hafner G (2005) Comparison of the platelet concentrate collection system with the plasmarich-in-growth-factors kit to produce platelet-rich plasma: a technical report. Int J Oral Maxillofac Implants 20(1):118-123

55. Arshdeep, Kumaran MS (2014) Platelet-rich plasma in dermatology: Boon or a bane? Indian J Dermatol Venereol Leprol 80:5-14

56. Arora NS, Ramanayake T, Ren YF, Romanos GE (2009) Plateletrich plasma: a literature review. Implant Dent 18(4):303-310 